\title{
The application of Image Kurtosis in Evaluation Method of Jamming Effect on ISAR
}

\author{
Cui Rui, Pan Jifei, Huang Jianchong \\ Electronic Engineering Institute \\ Hefei, china \\ Cuirui2002@sohu.com
}

\begin{abstract}
Some methods used to evaluate the jamming effect of common radar are unsuitable to ISAR because of its new work system. By comparing the change of image kurtosis, a novel evaluation method of jamming effect on ISAR Based on the kurtosis is presented in the paper, which can reflect the impact on the target image gray by the jamming. The method not only can be computed easily, but also has the merit of unitary. Furthermore, Two typical noise barrage jamming modes are carried out in the paper, the results of simulation prove the method is corrective and effective.
\end{abstract}

Keywords : ISAR; barrage jamming; kurtosis; evaluation;

\section{INTRODUCTION}

The Inverse Synthetic Aperture Radar is a high resolution imaging radar, since it has the ability to reconstruct a two dimension highresolution image of non-cooperative moving target, such as space objects, air targets and ships; it has been widely used for target classification, recognition and identification in military field [1]-[2]. As a main part of Electronic Counter -measures (ECM), Evaluation method of jamming effect on ISAR attracts much more attention. In [3]-[5], the change of the ISAR image index, entropy and the correlation of the ISAR image before and after jamming are used to analyze the jamming effect respectively. In [6], jamming effect which uses ENL (equivalent number of looks) is analyzed from image standard. Those methods can not become the uniform standard to evaluate the jamming effect on ISAR because of their different meaning.

By comparing the change of image Kurtosis, a novel evaluation method of jamming effect on ISAR is presented in the paper, which can reflect the impact on the imaging quality by the jamming.

\section{II . THE EVALUATION METHOD OF JAMMING EFFECT ON ISAR}

The main function of ISAR is target recognition, and that the recognition probability is closely linked with image quality. Commonly, the image of ISAR should has ability to distinguish neighborhood scatter points, also should keep tiny features of target and abundant gray levels. On the condition of being jammed, the target image will become illegible with the increase of jamming, at the same time, the distributing of image grey become more closed to Gaussian distribution. In order to describe this change quantitatively, the Kurtosis of image is introduced to evaluate the jamming effect on ISAR.

The Kurtosis is a very important high-rank statistic index, which can describe the distribution of image grey. It can be determined as:

$$
\operatorname{kurt}[f(x, y)]=\frac{E\left[f^{4}(x, y)\right]}{E^{2}\left[f^{2}(x, y)\right]}
$$

$f(x, y)$ represent the image grey, and it is not negative.

The Kurtosis is a number value that can 
reflect whether the data is consistent with Gaussian distribution. If $\operatorname{kurt}[f(x, y)]=0$, the image grey is consistent with Gaussian distribution. And if $\operatorname{kurt}[f(x, y)]$ is bigger, the data is far away from Gaussian distribution. The similarity of Kurtosis is introduced, which has the merit of unitary. It can be defined as follows:

$$
Q_{\text {kurt }}=\frac{2 \text { kurt }_{1} \cdot \text { kurt }_{0}}{\left(k u r t_{1}\right)^{2}+\left(k u r t_{0}\right)^{2}}
$$

Here $_{\text {kurt }}$ is the Kurtosis of ISAR image before jamming, kurt $_{1}$ is the Kurtosis of ISAR image after jamming, we can know that the range of the value of $Q_{k u r t}$ is $[0,1]$.if and only if the ISAR image is unchanged before and after jamming, $Q_{\text {kurt }}=1$. The similarity of Kurtosis became smaller and smaller with the increase of JSR. According to the above analysis, we know that the jamming effect on ISAR can be evaluated by the value of $Q_{k u r t}$. With the same JSR, if $Q_{k u r t}$ is smaller, then the jamming effect is better.

\section{THE SIMULATION OF JAMMING EFFECT ON}

ISAR

The ideal plan point target model is adopted to carry out the simulation of barrage jamming on ISAR in the paper. The parameters of system are represented as follows: Signal carrier frequency $\mathrm{f0}=10 \mathrm{GHz}$, bandwidth $\mathrm{B}=200 \mathrm{MHz}, \quad \mathrm{PRF}=200$ $\mathrm{HZ}$, the radial velocity of plan $\mathrm{v}=15 \mathrm{~m} / \mathrm{s}$. Noise AM jamming and noise FM jamming are carried out in the paper. The signal of noise AM can be described as ${ }^{[7]}$ :

$$
J(t)=\left(U_{0}+u_{n}(t)\right) \cos \left(2 \pi f_{0} t+\varphi\right)
$$

Where modulated noise $u_{n}(t)$ is a wide-sense stationary stochastic processes, its mean is zero and variance is $\sigma_{n}^{2}$, amplitude of carrier wave is $U_{0}$, carrier frequency is $f_{0}$, original phase of carrier wave is $\varphi$.The signal of noise FM can be expressed as:

$$
J(t)=U_{0} \cos \left[2 \pi f_{j} t+2 \pi k_{F M} \int_{0}^{t} u(\tau) d \tau+\phi\right]
$$

Where modulated noise $u(t)$ is a wide-sense stationary stochastic processes, its mean is zero and variance is $\sigma_{n}^{2}$, amplitude of carrier wave is $U_{0}$, carrier frequency is $f_{i}$, original phase of carrier wave is $\varphi$, coefficient of frequency modulation is $k_{F M}$.

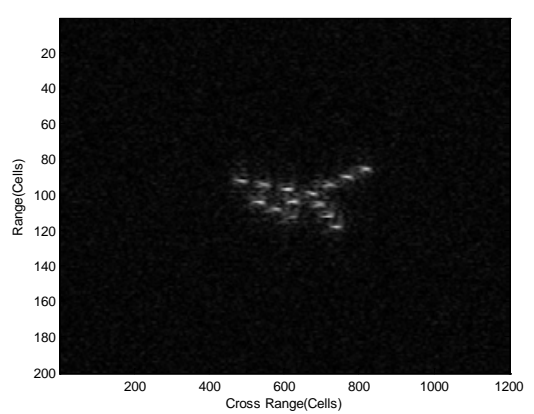

(a) $\mathrm{JSR}=5 \mathrm{~dB}$

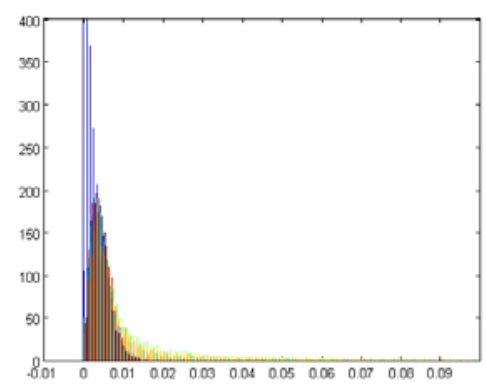

(b) $\mathrm{JSR}=5 \mathrm{~dB}$

Fig1 the target grey image and the grey of image

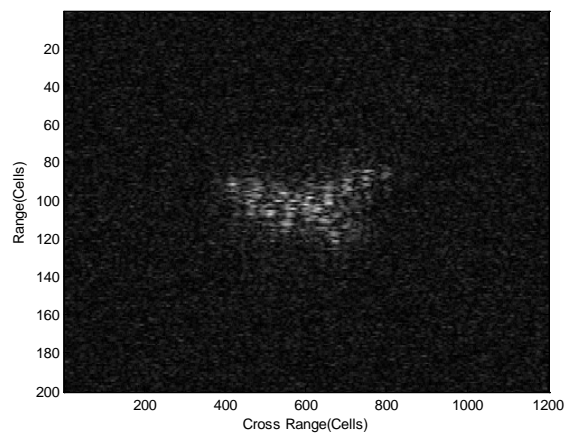

(a) $\mathrm{JSR}=10 \mathrm{~dB}$

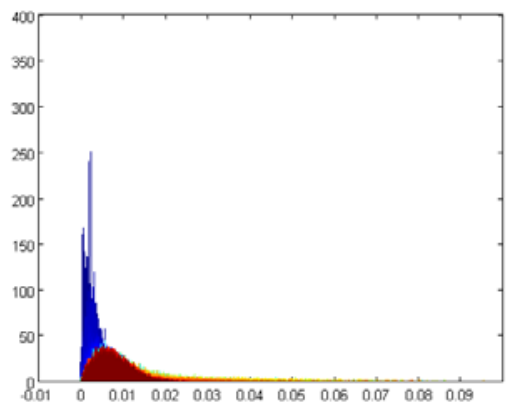

(b) $\mathrm{JSR}=10 \mathrm{~dB}$

Fig2 the target grey image and the grey of image

With the different JSR, the target grey image and the grey of image that jammed by FM noise are given respectively as Fig1, Fig2. The quality of image decline with the increasing of JSR and the image is destroyed more badly. We can also get that the distributing of image grey become more closed to Gaussian distribution. 


\section{IV . QUANTITATIVE JAMMING EFFECT EVALUA} -TIONS

According to formula (3) and (4), the jamming simulation is carried out respectively, the Kurtosis and the similarity of Kurtosis of image of two images can be calculated according to formula (1) and (2). The relationship between JSR can be shown as Fig.3 and Fig.4:

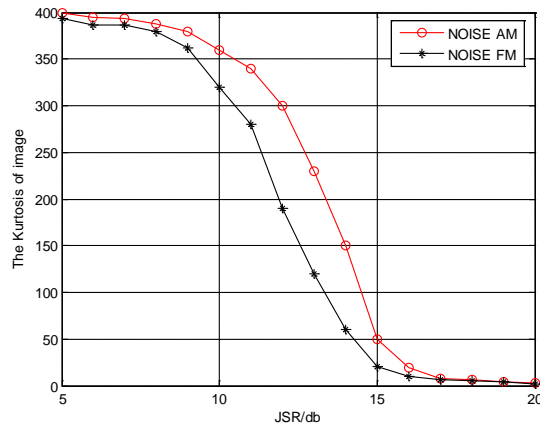

Fig3 curve of the Kurtosis

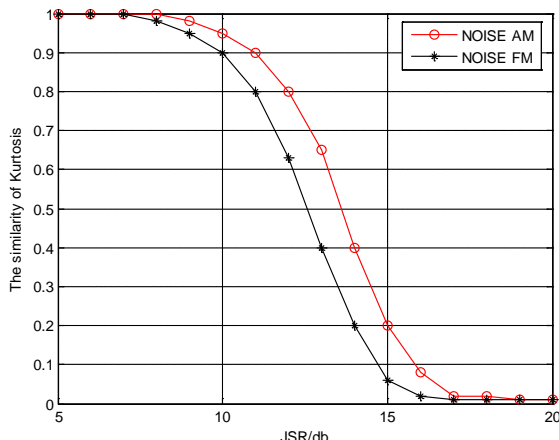

Fig4 curve of the similarity of Kurtosis

The conclusions can be got from the Fig.3 and Fig.4:

1 . With the increasing of JSR and the image is destroyed more badly. The distributing of image grey becomes more closed to Gaussian distribution.

2. The evaluation method of jamming effect that using the similarity of Kurtosis has the merit of unitary.

3. With the same JSR, the Kurtosis and the similarity of Kurtosis of image that jammed by noise FM is smaller than that jammed by noise AM. It proves that the jamming effect of noise FM is better than noise AM, which is consistent with Fig1 and Fig 2.

4. Two curves get together when JSR is bigger than $15 \mathrm{~dB}$, which mean the image have been jammed badly and the noise have made evaluation criterion invalidated.

\section{CONClusions}

The quantitative results that Fig.4 gives is consistent with the straight results that Fig. 1 and Fig.2give, that is to say the jamming effect of noise FM are better than the effect of noise AM in the same JSR. Both curves reflect clearly distortion degree of the jammed target during the whole jamming process, so the evaluation method of jamming effect that the paper proposes is proved to be true and effective. Besides, the method also has such merits as unitary and computed easily. The evaluation method of deceive jamming effect on ISAR based on the Kurtosis will be the main research direction in the future.

\section{REFERENCES}

[1] CHEN C C. Target-Motion-Induced Radar Imaging [J]. IEEE Trans on AES, 1980, 16(1):15-22

[2] Bao zheng, Xing Mengdao, WangTong. Technology of radar imaging. Publishing house of Electronic Industry, 2005

[3] Zhang Xiaole, "The research on the SAR jamming and jamming effect evaluating", Master's dissertation, Chengdian University, 2006.

[4] Wang Zhen-nan, Jia Xin, Wu Yan-Hong etc, "ISAR jamming effectiveness evaluation method based on information entropy,"Foreign electronic measurement technology.vol.27,no.9,2008,pp.17-20.

[5] Li Yuan, Chen Huilian, "Evaluation method of jamming effect on ISAR Based on Correlation Coefficient," Journal of University of Electronic Science and Technology, vol.35,no.4,Aug.2006,pp.468 -470.

[6] Cui Rui, Xue Lei, Wang Bo, "Evaluation method of jamming effect on ISAR Based on Equivalent number of looks," Systems Engineering and Electronics. vol.30,no.5,2008,pp.887-888.

[7] LENG Chuan-hang, FU YU-Sheng, PI Yi-Ming, HOU Yin-ming. Research on simulation of interference on inverse synthetic aperture $\operatorname{radar}[\mathrm{J}]$. the technology of electronic warfare, 2005, (5) : 35-39.

[8] MIAO Yan-hong, ZHAO Guo-qing. Measurement of the jamming effect of synthetic aperture radar [J]. The technology of electronic warfare, 2004, 19 (4) : 19-25.

[9] Rafael C. Gonzalez, Richard E.Woods, "Digital Image Processing Second Edition," Publishing House of Electronics Industry, 2008

[10] Zhou Chen, "The teconolgy of evaluating jamming effect on ISAR ," Master's dissertation, Chengdian University, 2009 